\title{
Accurate High Frequency Lossy Model of Differential Signal Line Including Mode-conversion and Common-mode Propagation Effect
}

\author{
Seungyong Baek, Seungyoung Ahn, Jongbae Park, \\ and Joungho Kim \\ Terahertz Interconnection and Package Laboratory, \\ Division of Electrical Engineering, \\ Department of EECS, KAIST \\ 373-1 Kusong-dong, Yusong-gu, Daejon 305-701, KOREA \\ bujirun@eeinfo.kaist.ac.kr, teralab@ee.kaist.ac.kr
}

\author{
Jonghoon $\mathrm{Kim}^{*}$ and Jeong-hyeon Cho* \\ *Module Design Group, Memory Division \\ Samsung Electronics Co., LTD \\ San \#16 Banwol-Ri, Taean-Eup, Hwasung-City, \\ Gyeonggi-Do, KOREA. \\ jh9.kim@samsung.com, caleb1@samsung.com
}

\begin{abstract}
The loss of multi-layer printed circuit board (PCB) becomes a crucial problem and lacks precise models in highspeed interconnections such as SerDes channel. Moreover, unbalanced and discontinuous structures generate undesirable mode-conversion, differential-to-common mode and common-todifferential mode. In this paper, we have proposed an accurate and efficient model of differential line where all of the modeconversion, common-mode propagation and frequencydependent loss are taken into consideration over $\mathrm{GHz}$ frequency range.
\end{abstract}

Kegwords-component; differential line model, mode-conversion, frequency dependent loss.

\section{INTRODUCTION}

Recently, differential signaling has become a popular interconnection scheme for high-speed digital applications such as FiberChannel, Infiniband, OIF, RapidIO, SerialATA and XAUI [1]. This is because the differential signaling does not only reduce crosstalk and electromagnetic interference (EMI) noise problem, but also enhances noise immunity against common-mode power/ground noises [2]. To take these advantages of differential signaling over $\mathrm{GHz}$, differential lines need to be carefully designed. Its impedance should be well controlled all the way from transmitter to receiver so that signal distortion, delay and skew are kept within acceptable margin. Furthermore, the two lines of a differential pair should be balanced.

However, the electrical parasitic elements from connector, package, via, bond pad, and device input ESD capacitors cause the unbalance and discontinuity of differential lines in PCB. As the data rate of the serial communication exceeds over $\mathrm{GHz}$ range, this becomes more crucial problem hurting the signal integrity. This is because the unbalanced and discontinuous structures generate undesirable mode-conversion (differentialmode to common-mode or common-mode to differentialmode) and differential-mode signal converted from commonmode noise makes the noise immunity wrong against common- mode power/ground noise. Furthermore, the power loss in differential lines also becomes a critical problem at high frequency range over $\mathrm{GHz}$, which is resulted from increased the skin effect loss and dielectric loss [3]. Therefore, it is very important to provide precise and reliable lossy differential line model considering mode-conversion and common-mode propagation effect and to predict loss and mode-conversion effect.

In this paper, we propose an accurate and efficient model of differential line where all of the mode-conversion, commonmode propagation and frequency-dependent loss are taken into consideration. In the proposed modeling procedure, $S_{11}$ parameters of mixed-mode S-parameters, $S_{\mathrm{ddl1}}, S_{\mathrm{dc} 11}, S_{\mathrm{cd} 11}$ and $S_{\mathrm{ccl} \text {, }}$, are measured from open and short patterned differential lines, and equivalent circuit model parameters are extracted from a subsequent de-embedding and fitting process for frequencies up to $3 \mathrm{GHz}$. We also verified the proposed model by observing the common-mode output voltages using timedomain measurement.

\section{DiFFERENTIAL LINE MODEL CONSIDERING MODE- CONVERSION}

Since differential line consists of two conductors, two modes exist according to shape of field; one is odd-mode and the other is even-mode. Moreover, mode-conversions are also existed on the unbalanced and discontinuous structures. We can obtain odd-mode, even-mode, and mode-conversion circuit model parameters using differential-mode S-parameter, common-mode S-parameter, and mode-conversion Sparameters of mixed-mode S-parameter.

$$
\left(\begin{array}{llll}
S_{d d 11} & S_{d d 12} & S_{c c 11} & S_{d c 12} \\
S_{d d 21} & S_{d d z 2} & S_{c k 21} & S_{d c 22} \\
S_{c d 11} & S_{c d 12} & S_{c c 11} & S_{c c 12} \\
S_{c d 21} & S_{c d \geq 2} & S_{c c 21} & S_{c c 22}
\end{array}\right)=M\left(\begin{array}{llll}
S_{11} & S_{12} & S_{13} & S_{14} \\
S_{21} & S_{22} & S_{23} & S_{24} \\
S_{31} & S_{32} & S_{33} & S_{34} \\
S_{41} & S_{42} & S_{43} & S_{44}
\end{array}\right) M^{-1}
$$




$$
M=\frac{1}{\sqrt{2}}\left(\begin{array}{cccc}
1 & -1 & 0 & 0 \\
0 & 0 & 1 & -1 \\
1 & 1 & 0 & 0 \\
0 & 0 & 1 & 1
\end{array}\right)
$$

Mixed-mode S-parameter was proposed by David. E. Bockelman in 1995 [4]. He extended a concept of the existing S-parameter to apply to differential circuit. In general, a differential circuit responds to both a differential-mode and a common-mode. Therefore, mixed mode S-parameter includes the differential-mode common-mode and any mode-conversion responses. As shown in the above matrix, mixed-mode $\mathrm{S}$ parameter is easily obtained through simple equation. Each partition represents a two-by-two S-parameter submatrix. The partitions labeled $S_{d d}$ are the differential-mode S-parameter, $S_{c c}$ are the common-mode S-parameter, and $S_{d c}$ and $S_{c d}$ are the mode-conversion S-parameter. Where $S_{\mathrm{dc}}$ describes the conversion of common-mode waves into differential-mode waves and $\mathrm{S}_{\mathrm{cd}}$ describes the conversion of differential-mode waves into common-mode waves.

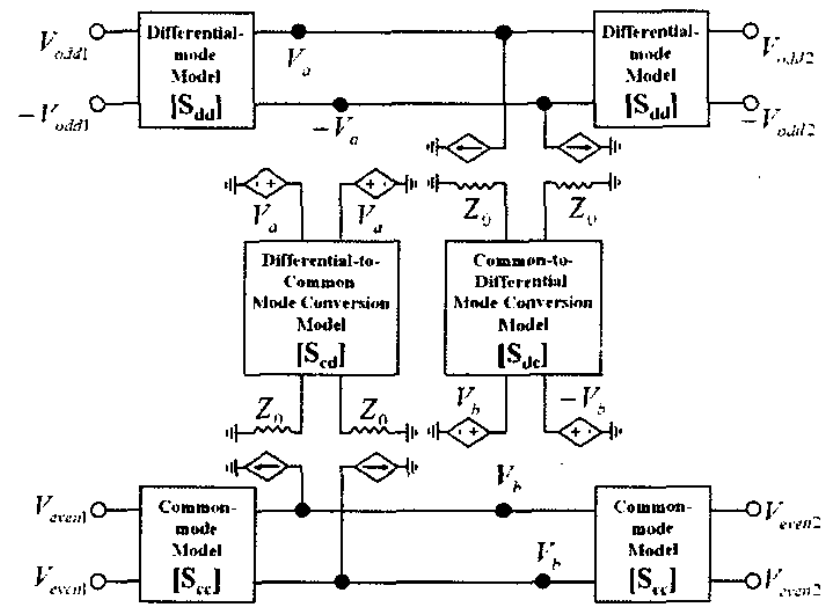

Figure 1. Block diagram about basic concept of differential line model considering mode conversion.

Figure 1 shows the block diagram about basic concept of the proposed differential line model considering mode conversion. The purely differential-mode parameter is modeled as odd-mode circuit model using differential-mode S-parameter, $\mathrm{S}_{\mathrm{dd}}$, and the purely common-mode parameter is modeled as even-mode circuit model using common-mode $\mathrm{S}$-parameter, $\mathrm{S}_{\mathrm{cc}}$. The differential-to-common mode conversion parameter is modeled using differential-to-common mode S-parameter, $\mathrm{S}_{\mathrm{cd}}$, and the common-to-differential mode conversion parameter is modeled using common-to-differential mode S-parameter, $S_{d c}$. The voltage controlled voltage source induces the input voltage of mode-conversion model from odd-mode model and evenmode model. An amount of mode-conversion is added into odd-mode model and even-mode model using the voltage controlled current source. Using controlled source, the models for purely differential-mode and common-mode are not influenced by mode-conversion model, because modeconversion models are not directly connected into the oddmode model and even-mode model. The modeling procedure to obtain each circuit model parameters of figure 1 is shown in figure 2 . The proposed modeling procedure is based on 2-port S-parameter measurement using 2-port vector network analyzer (VNA). But, it is hard to measure differential line using 2-port VNA, because the differential line is 4-port network. However, if open and short patterned differential lines are measured, it is possible to extract all of differential-mode, common-mode and mode-conversion model parameters.

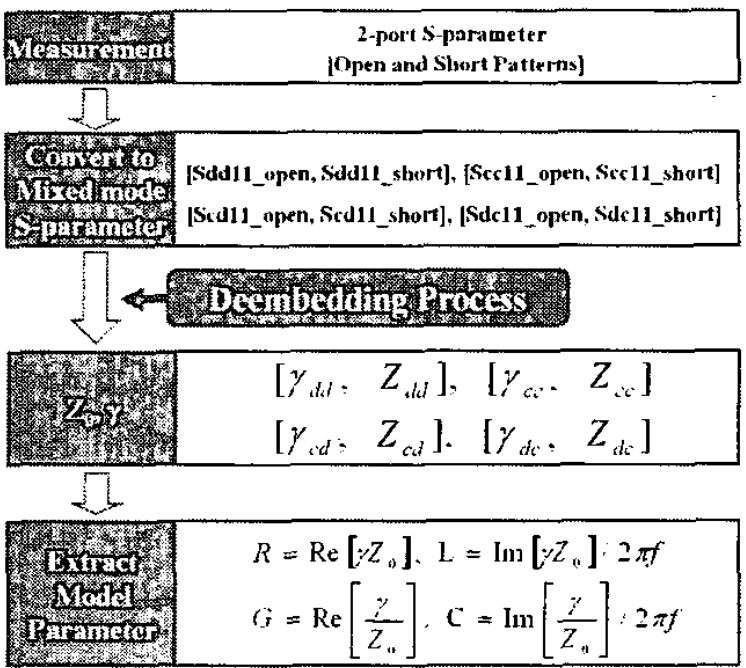

Figure 2. Proposed measurement based modeling procedure.

The measured 2-port S-parameters of open and short patterned differential line are converted into $S_{11}$ parameters of

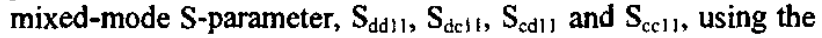
equation (1)-(4). In fact, it is difficult to embody the perfect open and short circuits on PCB because of the capacitance by fringing field at open point and the inductance by via for short pattern. Therefore, these capacitance and inductance have to be de-embedded from the measured S-parameter. We can deembed the unwanted parasitic factors and extract propagation constant and characteristic impedance of differential-mode, common-mode, and mode-conversion using the proposed deembedding process as shown in figure 3 . From these results, model parameters, R, L, G, and $C$, are acquired by simple equations which are shown in bottom box of figure 2 .

$$
\begin{aligned}
& S_{d d d 1}=\frac{1}{2}\left(S_{11}-S_{21}-S_{12}+S_{22}\right) \\
& S_{d c k 11}=\frac{1}{2}\left(S_{11}-S_{21}+S_{12}-S_{22}\right) \\
& S_{c d 11}=\frac{1}{2}\left(S_{11}+S_{21}-S_{12}-S_{22}\right) \\
& S_{c c 11}=\frac{1}{2}\left(S_{11}+S_{21}+S_{12}+S_{22}\right)
\end{aligned}
$$


As mentioned above, since our proposed modeling method is based on measurement of open and short patterned differential lines, the parasitic capacitance and inductance which are generated by open and short pattern have to be deembedded from the measured S-parameter. Figure 3 shows the concept of de-embedding process.

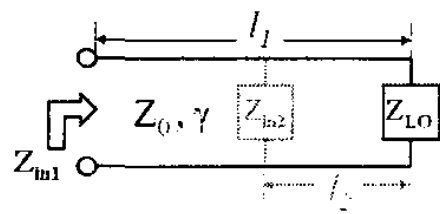

(a)

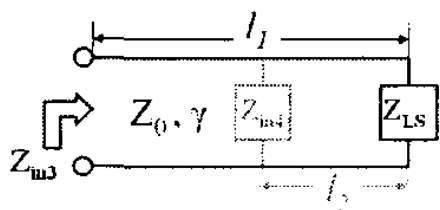

(c)

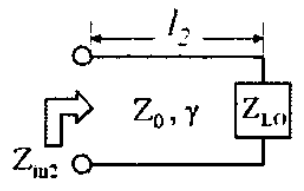

(b)

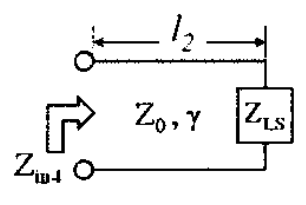

(d)
Figure 3. Concept of deembedding process (a) Transmission line with input impedance $Z_{\text {inl, }}$, open load $Z_{\mathrm{LO}}$ and length $I_{t}$ (b) Transmission line with input impedance $Z_{i n 2}$, open load $Z_{L O}$ and length $I_{2}$ (c) Transmission line with input

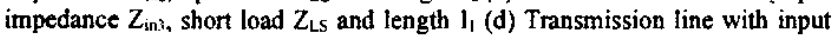
impedance $Z_{\mathrm{inH}}$, open load $Z_{\mathrm{LS}}$ and length $\mathrm{l}_{2}$

As shown in Figure 3, the two kinds of open and short pattern are measured. In ideal case, the impedance of open load $Z_{\mathrm{LO}}$ is infinite quantity and short load $Z_{\mathrm{LS}}$ is zero. However, $Z_{\mathrm{LO}}$ and $Z_{\mathrm{LS}}$ have arbitrary impedance. Firstly, we measured $Z_{\text {in2 } 2}$ and $Z_{\text {int }}$ which are input impedances of transmission line located at $l_{2}$ from $Z_{\mathrm{LO}}$ and $\mathrm{Z}_{\mathrm{LS}}$, and then Figure 3 (a) and (c) are able to be changed into the transmission lines which have length of $l_{1}-l_{2}$ and load impedance of $Z_{\text {in } 2}$ and $Z_{\text {in } 4}$, respectively. $Z_{1 n !}$ and $Z_{i n 3}$ are calculated by the equation (S) and (6).

$$
\begin{aligned}
& Z_{i n 1}=Z_{01} \frac{Z_{i n 2}+Z_{0} \tanh \gamma\left(l_{1}-l_{2}\right)}{Z_{0}+Z_{i n 2} \tanh \gamma\left(l_{1}-l_{2}\right)} \\
& Z_{m 3}=Z_{01} \frac{Z_{i n 4}+Z_{01} \tanh \gamma\left(l_{1}-I_{2}\right)}{Z_{01}+Z_{i n 4} \tanh \gamma\left(l_{1}-I_{2}\right)}
\end{aligned}
$$

Since $Z_{\text {in } 1}, Z_{\text {in } 2}, Z_{\text {in:3 }}$, and $Z_{\text {in } 4}$ are able to be known from measured S-parameters, the characteristic impedance and the propagation constant can be derived from the equation (7) and (8).

$$
\begin{gathered}
Z_{0}=\sqrt{\frac{\left(Z_{i n 3} \cdot Z_{m 4}\right)\left(Z_{m 1}-Z_{m 2}\right)-\left(Z_{m 1} \cdot Z_{m 2}\right)\left(Z_{m, 3}-Z_{m 4}\right)}{\left(Z_{m 1}-Z_{m 2}\right)-\left(Z_{m 3}-Z_{m 4}\right)}} \\
\gamma\left(l_{1}-I_{2}\right)=\tanh ^{-1}\left(\frac{Z_{0}\left(Z_{m n 1}-Z_{m 2}\right)}{Z_{0}^{2}-Z_{i m 1} \cdot Z_{m 2}}\right)
\end{gathered}
$$

From these results, model parameters, R, L, G, and C, of each mode are extracted.

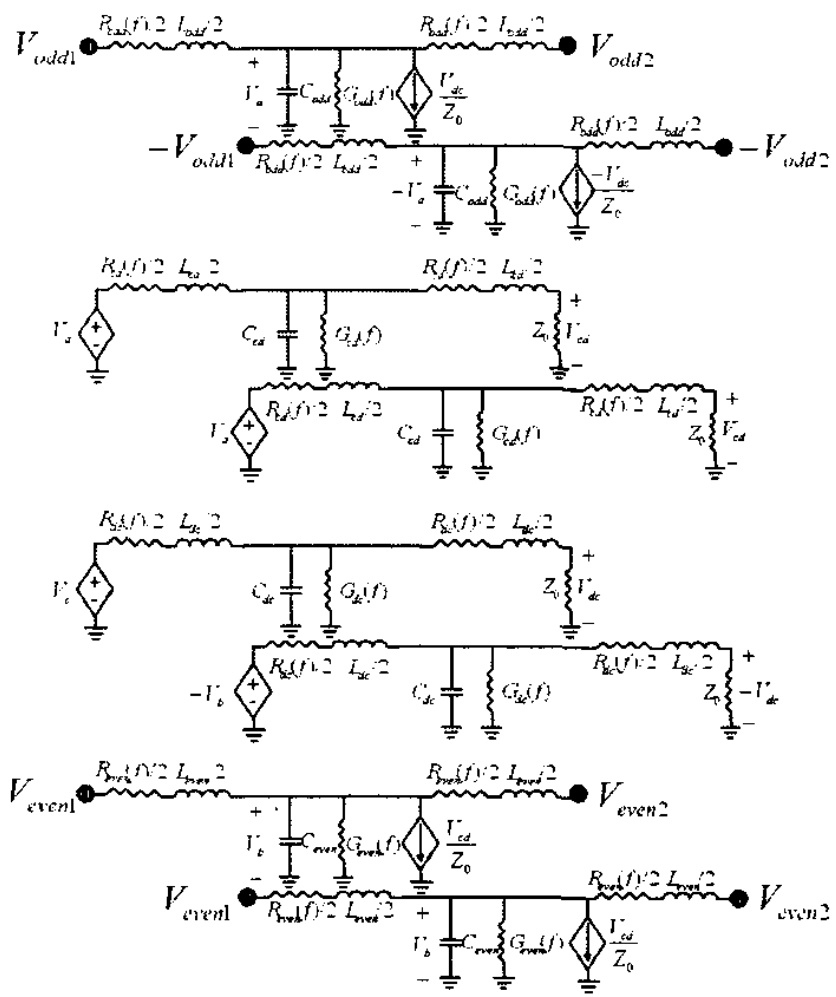

Figure 4. Circuit model of differential line considering differential-tocommon mode conversion

Figure 4 shows the proposed circuit model of differential line considering mode-conversion. As shown in Figure 1, differential-mode and common-mode is modeled as odd-mode and even-mode model using $S_{\mathrm{dd} 11}$ and $S_{\mathrm{cc} 11}$ of open and short patterns, and mode-conversion is modeled by $S_{\mathrm{dc} ! 1}$ and $S_{\mathrm{cd} 11}$. The controlled sources induce voltage into mode-conversion model and correspond to additional current due to modeconversion.

\section{MEASUREMENT}

In order to apply the proposed modeling procedure and the circuit model, we selected pattern which is unbalanced differential line. The unbalanced differential line has 250 um and $350 \mathrm{um}$ trace width respectively. For de-embedding the capacitance by fringing field at open ended and the inductance by via for short pattern, two open and short patterned differential lines which have a different length are measured as shown in Figure 5. We extracted the circuit model parameters of each mode, differential-mode, common-mode, and modeconversion, through S-parameter measurement and proposed modeling procedure. The extracted model parameters are shown in Figure 6 and Figure 7.

Figure 6 shows odd-mode and even-mode circuit model parameters which are obtained by $S_{\mathrm{ddl}}$ and $S_{\mathrm{ccll}}$ of open and 
short patterned differential line. It is certificated that the resistance and the conductance are frequency dependent parameters, and the inductance and the capacitance hardly depend on frequency. The resistance and the conductance are fitted by frequency function from equation (9) to (12), and we selected the optimized inductance and capacitance which are $1.37 \mathrm{nH}$ odd-mode inductance, $2.04 \mathrm{nH}$ even-mode inductance, $0.56 \mathrm{pF}$ odd-mode capacitance, and $0.46 \mathrm{pF}$ even-mode capacitance, respectively.

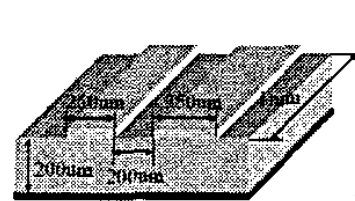

(a)

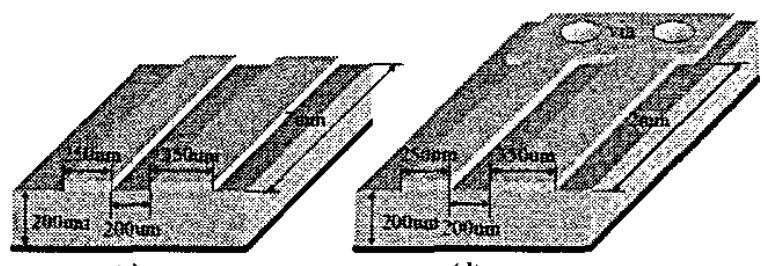

(c)

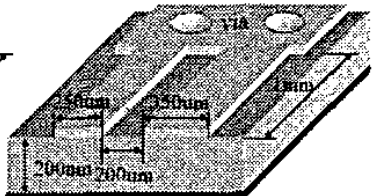

(b)

(d)

Figure 5. Dimension of measured DUT which are $250 \mathrm{um}, 350 \mathrm{um}$ width $200 \mathrm{um}$ space and $200 \mathrm{um}$ height. (a) Open pattemed differential line with $1 \mathrm{~mm}$ length (b) Short patterened differential line with $1 \mathrm{~mm}$ (c) Open patterned differential line with $2 \mathrm{~mm}$ (d) Short patterend differenetial line with $2 \mathrm{~mm}$

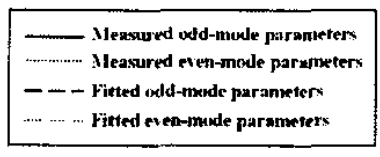

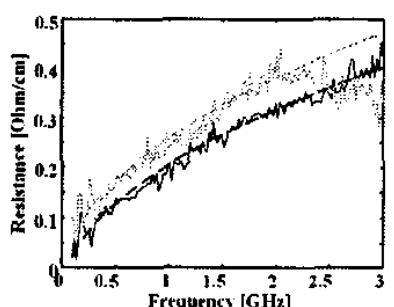

(a)

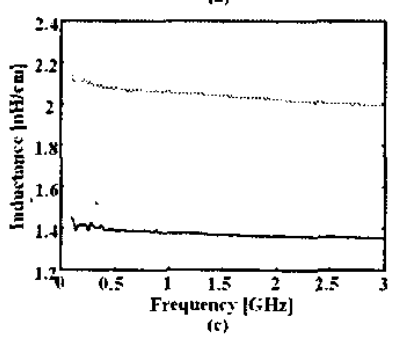

Figure 6. Odd-mode and even-mode circuit model parameter using mixed mode S-parameter (a) Odd-mode and even-mode resistance per $\mathrm{cm}$ (b) Oddmode and even-mode conductance per $\mathrm{cm}$ (c) Odd-mode and even-mode inductance per $\mathrm{cm}$ (d) Odd-mode and even-mode capacitance per $\mathrm{cm}$.

$$
\begin{aligned}
& R_{\text {redid }}=0.85 \times 10^{-5} \sqrt{f} \\
& R_{\text {eren }}=0.92 \times 10^{-5} \sqrt{f} \\
& G_{\text {cxdl }}=8.13 \times 10^{-14} f \\
& G_{e v e n}=5.84 \times 10^{-14} f
\end{aligned}
$$

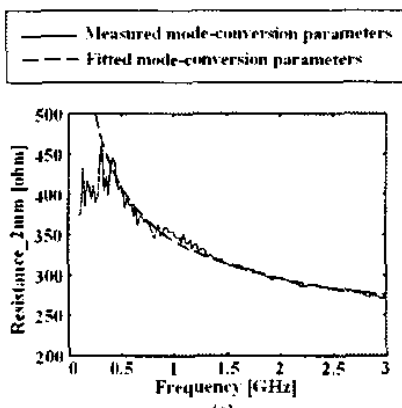

(a)
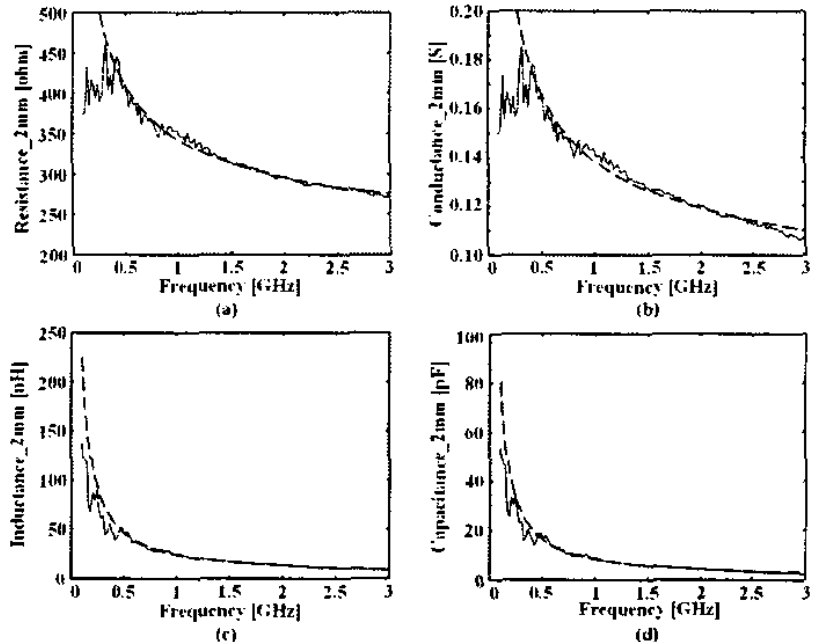

Figure 7. Mode-conversion circuit model parameter using mode-conversion 2-port S-parameter of mixed mode S-parameter (a) Mode-converson resistance of $2 \mathrm{~mm}$ (b) Mode-conversion conductance of $2 \mathrm{~mm}$ (c) Modeconversion inductance of $2 \mathrm{~mm}$ (d) Mode-conversion capacitance of $2 \mathrm{~mm}$.

$$
\begin{aligned}
& R_{c d}=182.35+5.1 \times 10^{6} / \sqrt{f} \quad[\mathrm{ohm}] \\
& G_{c d}=0.0722+2.1 \times 10^{3} / \sqrt{f} \quad[S] \\
& L_{c d}=5.24 \times 10^{-9}+17.5 / f \quad[\mathrm{H}] \\
& C_{c d}=0.135 \times 10^{-32}+0.008 / f \quad[F]
\end{aligned}
$$

Figure 7 shows model parameters of differential-tocommon mode-conversion, all of mode-conversion circuit model parameters depend on the frequency. The resistance and the conductance are inversely proportional to square root frequency such as (13) and (14). The inductance and the capacitance are inversely proportional to frequency such as (15) and (16). From four parameters, R, L, G, and C, of modeconversion, we can explain the phenomenon that modeconversion is generated more and more at higher frequency. Since model parameters of common-to-differential modeconversion were obtained the similar results to those of differential-to-common mode-conversion, the model parameters of equation (13)-(16) were also applied to commonto-differential mode-conversion. 
From these results, the extracted parameters by measured results and the fitted results are close each other up to $3 \mathrm{GHz}$. We can certificate that the proposed equivalent circuit model agrees well with the extracted parameters from the S-parameter measurement.

In order to verify the proposed model and the extracted model parameters, we measured time-domain common-mode output signal as shown in Figure 8. The differential-mode input voltage which has peak-to-peak $400 \mathrm{mV}$ and $500 \mathrm{MHz}$ clock frequency was applied to differential line, and transmitted common-mode voltages were measured which were obtained by sum of transmitted differential-mode voltages. And we used unbalanced differential line with $10 \mathrm{~cm}$ length which has $250 \mathrm{um}$ and $350 \mathrm{um}$ width respectively.

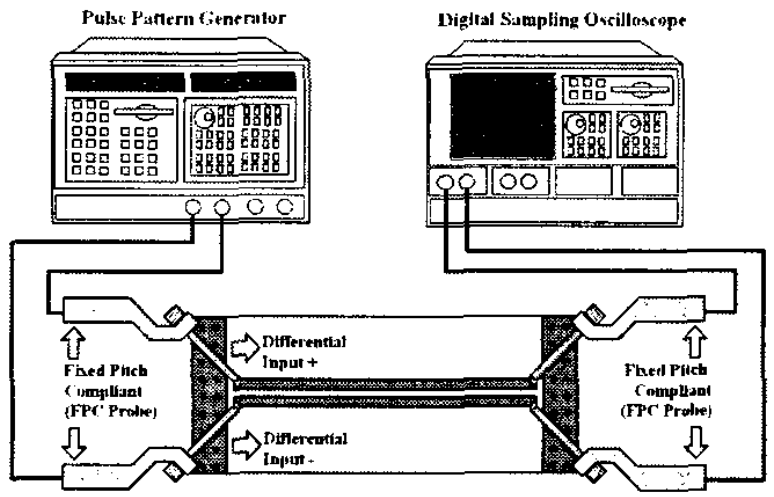

Figure 8. Time-domain measurement setup for evaluation of the proposed model considering mode-conversion.

Finally, we obtained transmitted common-mode-output voltage as shown in Figure 9. The solid line is the measured common-mode output voltage by setup of figure 8 , and the dashed line indicates the common-mode output voltage obtained by proposed model. Since the differential input signals generated by pulse pattern generator have small skew, the voltage peaks exist in the measured result where rising and falling of two differential signals cross. As shown in Figure 9, common-mode output voltage generated peak-to-peak $16 \mathrm{mV}$ at each differential line. It means $4 \%$ of input voltage. It was certificated that the common-mode output voltage obtained from the proposed equivalent circuit model agrees well with the measured common-mode output voltage.

\section{CONCLUSION}

It has been demonstrated an accurate and efficient model of differential line where all of the mode-conversion, commonmode propagation and frequency-dependent loss are taken into consideration. In the proposed modeling procedure, $S_{11}$ parameters of mixed-mode S-parameters are measured from open and short patterned differential lines, and equivalent circuit model parameters are extracted from a subsequent de- embedding and fitting process for frequencies up to $3 \mathrm{GHz}$. We also verified the proposed model by observing the commonmode output voltages. The common-mode output voltage obtained from the proposed equivalent circuit model also matches well with the measured common-mode output voltage. Applying the proposed modeling method to the model of differential channels, we can expect that it is easy to characterize mode-conversion generated by unbalanced and discontinuity structure. Moreover, our model facilitates that the properties of each mode, differential-mode and common-mode, are understood, because proposed model is divided into differential-mode model and common-mode model.

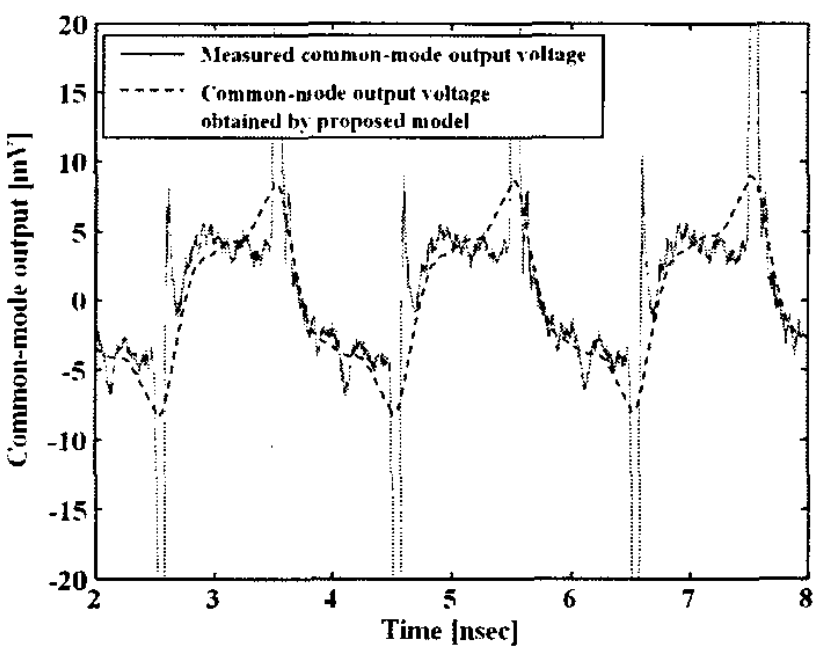

Figure 9. Common-mode output voltage obtained by sum of two differentialmode ouput voltages.

\section{ACKNOWLEDGMENT}

The authors would like to thank Sang Hoon Lee, who works in Samsung Advanced Technology Training Human Resource Team, for his support in the measurement of this study.

\section{REFERENCES}

[1] Henry Wu, Wendemagegnehu T. Beyene, Newton Cheng, Ching-Chao Huang, and Chuck Yuan, "Design and Verificaton of Differential Transmission Lines," Electrical Performance of Electronic Packaging, pp. 85-88, Oct 2001.

[2] David E. Bockelman and Wiliam R. Eisenstadt. "Direct Measurement of Crosstalk Between Integrated Differential Circuit." IEEE Trans. Microwave Theory Tech., vol. 48, no. 8, August 2000.

[3] A. Deutsch, "High-speed signal propagation on lossy transmission lines," IBM J. RES. Develop. Vol. 34, no. 4, July 1990.

[4] David E. Bockelman and Wiliam R. Eisenstadt. "Combined Differential and Common-Mode Scattering Parameters: Theory and Simulation." IEEE Trans. Microwave Theory Tech., vol. 43, no. 7, July 1995. 